\title{
An Evaluation Method of Underwater Ocean Environment Safety Situation Based on D-S Evidence Theory
}

\author{
Yuxin Zhao, Renfeng Jia, and Chang Liu \\ College of Automation, Harbin Engineering University, Harbin 150001, China \\ Correspondence should be addressed to Yuxin Zhao; zhaoyuxin@hrbeu.edu.cn
}

Received 15 December 2014; Accepted 29 January 2015

Academic Editor: Shaoqing Zhang

Copyright (c) 2015 Yuxin Zhao et al. This is an open access article distributed under the Creative Commons Attribution License, which permits unrestricted use, distribution, and reproduction in any medium, provided the original work is properly cited.

\begin{abstract}
Because of complex ocean environment, underwater vehicles are facing many challenges in navigation safety and precise navigation. Aiming at the requirements of underwater navigation safety, this paper presents an evaluation method of underwater ocean environment safety situation based on Dempster-Shafer (D-S) evidence theory. Firstly, the vital ocean environment factors which affect the underwater navigation safety are taken into account, and a novel basic probability assignment (BPA) construction method of ocean environment factors is proposed according to their characteristics. Then, a new transformation method of BPA to decisionmaking probability is put forward to deal with the uncertainty degree. Furthermore, the super-standard weight is applied to preprocess the BPA, and D-S combination rule is used to acquire the evaluation result by fusing the preprocessed BPA. Ocean environment safety situation index is obtained by quantizing the evaluation grades. Finally, experimental results show that the method proposed has the superior practicability and reliability in actual applications.
\end{abstract}

\section{Introduction}

Ocean is the main resource for human survival in the future. With the increasing demand for ocean development and military applications, the underwater vehicle is a powerful tool for human to explore and utilize ocean, which has become the field to which the world technological power (the United States, Britain, Japan, etc.) gives priority to develop [1]. In order to obtain more ocean interests, a large number of autonomous underwater vehicles (AUVs) and remotely operated vehicles (ROVs) are widely applied in ocean surveys, scientific experiments, and resource explorations. Whatever the purposes of underwater vehicles are, the navigation safety is the fundamental premise to make them complete various tasks effectively. The navigation safety is affected by many ocean environment factors, and it is closely related to the basic characteristics and distribution of ocean environment factors. Ocean environment factors are often the "doubleedged sword"; they not only can bring some advantages, but also can bring the challenges to underwater navigation safety. If ocean environment factors are well used, they can improve the operational effectiveness of underwater vehicles. Otherwise, they will threaten the navigation safety. Over the long-term explorations (including ocean surveys, ocean researches, the establishments of various ocean observation instruments, etc.), humans have a certain level of knowledge and ability to control ocean. But so far, they have not solved effectively and completely the problem of navigation safety of underwater vehicles in complex ocean environment. Even if underwater navigation technology has been quite advanced, navigation safety accidents of underwater vehicles happened frequently in recent years. The problem of underwater vehicles' navigation safety has become the topic of common concern.

Evaluating ocean environment (particularly underwater environment) safety situation is very important for underwater navigation safety, which can provide objective, accurate, and quantitative evaluation result and supply the basis for underwater vehicles to take advantage of ocean environment effectively. Although domestic and foreign scholars have acquired many achievements in underwater vehicles environment modeling theory [2-7], they almost paid no attention to ocean environment safety situation. This paper puts forward a method to evaluate ocean environment safety situation and tries to seek a scientific, rational, objective, and feasible solution for improving underwater navigation safety. 
Underwater navigation decision-making is a kind of intelligent behavior, which should be based on the whole situation description of ocean environment factors, rather than their accurate distribution results. In addition, ocean environment has strong randomness and uncertainty, which make it difficult to evaluate the safety situation. Therefore, evaluation method should be able to solve above problems effectively. D-S evidence theory $[8,9]$ has advantages and reliability in dealing with fuzzy and uncertain problems, which make it widely applied in many areas such as information fusion [10], fault diagnosis [11], and risk evaluation $[12,13]$. D-S evidence theory has profound theory foundation. It can surprisingly deal with uncertainty information and take full advantage of the multisource information to obtain better fusion results. Moreover, it has been proved that D$S$ evidence theory could achieve fast convergence without a priori probability and conditional probability [14]. So D$S$ evidence theory is used to evaluate the underwater ocean environment safety situation in this paper.

There are a lot of ocean environment factors which affect the underwater navigation safety. If all ocean environment factors are taken into account in the evaluation, which not only adds the complexity of evaluation model, but also may weaken the roles of main ocean environment factors, so it is required to follow the principles such as representative, independence, and feasibility. Based on these principles, this paper mainly discusses the influence of ocean current, tide current, and pycnocline on the underwater navigation safety.

In this paper, an efficient evaluation method of underwater ocean environment safety situation based on D-S evidence theory is presented to make underwater vehicles navigate safely. The next content is composed of three sections. In Section 2, the implementation process of evaluation method is introduced. In Section 3, experiments illustrate the effectiveness of the proposed method. Finally, the conclusion of this paper is described in Section 4.

\section{Materials and Methods}

The establishment of evaluation model based on D-S evidence theory includes constructing the BPA, dealing with the uncertainty degree, fusing the BPA, and obtaining the safety situation index. In this section, they will be illustrated in detail.

2.1. D-S Evidence Theory. D-S evidence theory is based on a nonempty set $\Theta$, called the frame of discernment (FOD). The elements in FOD $\Theta$ are mutually exclusive and exhaustive. A BPA is a mapping $m: 2^{\Theta} \rightarrow[0,1]$, satisfying

$$
\begin{gathered}
\sum_{A \subseteq \Theta} m(A)=1, \\
m(\emptyset)=0 .
\end{gathered}
$$

If $m(A)>0, A$ is called a focal element.

The lower bound for a set $A$, represented as $\operatorname{Bel}(A)$, is defined as follows:

$$
\operatorname{Bel}(A)=\sum_{B \subseteq A} m(B) .
$$

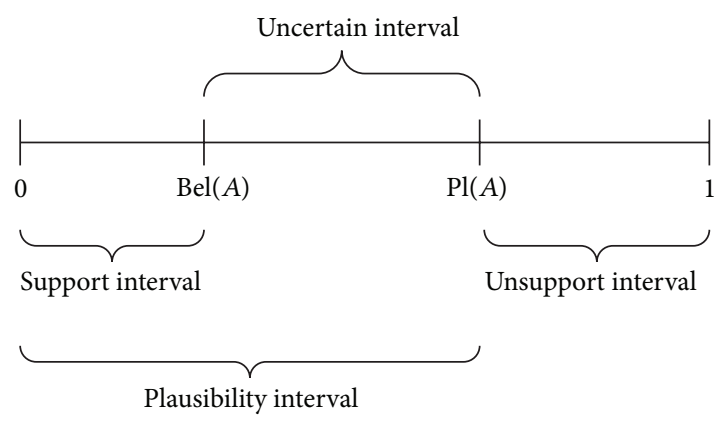

FIgURE 1: The relation between $\operatorname{Bel}(A)$ and $\operatorname{Pl}(A)$.

The upper bound for a set $A$, represented as $\operatorname{Pl}(A)$, is defined as follows:

$$
\operatorname{Pl}(A)=\sum_{A \cap B \neq \phi} m(B) .
$$

The relation between $\operatorname{Bel}(A)$ and $\operatorname{Pl}(A)$ can be seen from Figure 1.

$\mathrm{D}-\mathrm{S}$ evidence theory provides a very useful combination rule, denoted by $\left(m_{1} \oplus m_{2}\right)$ (also called the orthogonal sum of $m_{1}$ and $m_{2}$ ), which is defined as follows:

$$
m(A)=\frac{1}{1-k} \sum_{B \cap C=A} m_{1}(B) m_{2}(C),
$$

where

$$
k=\sum_{B \cap C=\phi} m_{1}(B) m_{2}(C)
$$

$k$ reflects the conflict between $m_{1}$ and $m_{2}$. Its role is to avoid nonzero probability being assigned to empty set during combination. D-S combination rule is both commutative and associative.

2.2. Constructing the BPA. The effective application of D-S evidence theory depends on rational construction of the BPA. However, how to construct the BPA is still an open issue. Currently, there are some researches in this field [15-23]. But the existing methods are not suitable for ocean environment factors. Therefore, this paper proposes a construction method of the BPA according to ocean environment factors' features.

First of all, the set of evaluation grades is denoted by $\Theta=\left\{e_{1}, e_{2}, \ldots, e_{N}\right\}$, and $N$ is the number of grades. Here we set $N=5,\left\{e_{1}, e_{2}, e_{3}, e_{4}, e_{5}\right\}$, representing "Very Safe (VS)", "More Safe (MS)", "General Safe (GS)", "More Dangerous (MD)", "Very Dangerous (VD)"\}, respectively, and corresponding risk intervals are represented in Table 1.

Afterwards, the BPA $m_{i, n}$ of $i$ th factor $f_{i}$ on $n$th evaluation grade $e_{n}$ is calculated separately according to the following two cases.

Case 1. If the value $x_{i}$ of ocean environment factor $f_{i}$ belongs to intervals VS, MS, GS, and MD, then $m_{i, n}$ is defined as

$$
m_{i, n}=\frac{\lambda}{\lambda+|| x_{i}\left|-\left(a_{n}+b_{n}\right) / 2\right|} .
$$


TABLE 1: The risk intervals.

\begin{tabular}{lcccrr}
\hline Factors & VS & MS & GS & MD & VD \\
\hline Ocean current $(\mathrm{m} / \mathrm{s})$ & {$[0.0,1.0]$} & $(1.0,2.0]$ & $(2.0,3.0]$ & $(3.0,4.0]$ & $(4.0,+\infty)$ \\
Tide current $(\mathrm{m} / \mathrm{s})$ & {$[0.0,0.6]$} & $(0.6,1.0]$ & $(1.0,1.5]$ & $(1.5,2.0]$ & $(2.0,+\infty)$ \\
Pycnocline $\left(\mathrm{kg} / \mathrm{m}^{4}\right)$ & {$[0.0,0.2]$} & $(0.2,0.4]$ & $(0.4,0.6]$ & $(0.6,0.8]$ & $(0.8,+\infty)$ \\
\hline
\end{tabular}

Case 2. If the value $x_{i}$ of ocean environment factor $f_{i}$ belongs to interval VD, then $m_{i, n}$ is defined as

$$
m_{i, n}=1 \text {, }
$$

where $a_{n}$ and $b_{n}$ represent the boundaries of each interval, and $\lambda=b_{n}-a_{n}$ is the adjustment parameter, which is used to ensure $m_{i, n} \in[0,1]$.

Due to the influence of uncertainty factors, there may be some uncertainty degree when calculating the BPA, which makes $m_{i, n}$ not necessarily equal to one. The following equation is used to represent the uncertainty degree:

$$
m(\Theta)=1-m_{i, n} .
$$

2.3. Dealing with the Uncertainty Degree. In order to obtain better evaluation result, $m(\Theta)$ need to be assigned to each evaluation grade, which requires transformation of decisionmaking probability. At present, there are a lot of transformation methods of decision-making probability [24-34], and these methods have certain advantages as well as some drawbacks. Based on the existing methods, for the sake of improving the shortcomings of Daniel's proportion transformation methods, this paper proposes a transformation method of decision-making probability based on the certainty degree. In accordance with the characteristics of BPA, the sum of each single proposition's BPA is regarded as the certainty degree of system, and the decision-making probability of each proposition is acquired by linear combination, which is the transformation method of decision-making probability based on proportional belief and proportional plausibility.

\subsubsection{Daniel's Proportional Transformations}

(1) Proportional Belief Transformation. Consider

$$
\operatorname{PropBelP}(A)=\sum_{A \subseteq X \in 2^{\Theta}} \frac{m(A)}{\sum_{B \in X} m(B)} m(X) .
$$

This method uses belief ratio between single proposition and multiple propositions to obtain the decision-making probability. The attitude of transformation is more optimistic, which is easy to increase the risk of decision-making.

(2) Proportional Plausibility Transformation. Consider

$$
\operatorname{PropPlP}(A)=\sum_{A \subseteq X \in 2^{\Theta}} \frac{\operatorname{Pl}(A)}{\sum_{B \in X} \operatorname{Pl}(B)} m(X) .
$$

This method uses plausibility ratio between single proposition and multiple propositions to obtain decision-making probability. The attitude of transformation is more conservative, which is not conducive to make decision.
2.3.2. Transformation Based on the Certainty Degree. In order to better take advantage of proportional transformation methods and overcome their deficiencies, this paper linearly combines these two approaches, which makes the attitude of transformation neither optimistic nor conservative, and draws reasonable decision-making probability.

Since single proposition's BPA reflects the precise level of system about the proposition, single proposition's BPA is lager, which indicates that the certainty degree of system about the proposition will be higher, and vice versa. Therefore, depending on the similar nature between the part and the whole, the sum of each single proposition's BPA can be regarded as the certainty degree of system and used as the weight to combine the proportional belief transformation method and proportional plausibility transformation method. When the certainty degree of system about the proposition is larger, the attitude of transformation should be relatively optimistic. On the contrary, it should be conservative.

Based on the foregoing, the transformation formula of new method is defined as follows:

$$
\begin{aligned}
P_{\text {new }}(A)= & \beta \cdot \sum_{A \subseteq X \in 2^{\Theta}} \frac{m(A)}{\sum_{B \in X} m(B)} m(X) \\
& +(1-\beta) \cdot \sum_{A \subseteq X \in 2^{\Theta}} \frac{\mathrm{Pl}(A)}{\sum_{B \in X} \mathrm{Pl}(B)} m(X),
\end{aligned}
$$

where

$$
\beta=\sum_{X \in 2^{\Theta},|X|=1} m(X)
$$

Since $A$ is single proposition, so formula (11) can be written as follows:

$$
\begin{aligned}
& P_{\text {new }}(A) \\
& =m(A)+\sum_{A \subseteq X \in 2^{\Theta},|X|>1}\left[\beta \cdot \frac{m(A)}{\sum_{B \in X} m(B)}\right. \\
& \left.+(1-\beta) \cdot \frac{\operatorname{Pl}(A)}{\sum_{B \in X} \operatorname{Pl}(B)}\right] m(X) .
\end{aligned}
$$

The effectiveness of new method can be illustrated by the following example.

Example 1. Suppose FOD $\Theta=\left\{\theta_{1}, \theta_{2}, \theta_{3}, \theta_{4}\right\}$; then, the BPA is given in Table 2 .

The results of new method compared to the other usual methods are shown in Table 3. 
TABLE 2: The BPA.

\begin{tabular}{lccccc}
\hline Propositions & $\theta_{1}$ & $\theta_{2}$ & $\theta_{3}$ & $\theta_{4}$ & $\theta_{1} \cup \theta_{2} \cup \theta_{3} \cup \theta_{4}$ \\
\hline$m$ & 0.8 & 0 & 0 & 0 & 0.2 \\
\hline
\end{tabular}

TABLE 3: The comparisons of different methods.

\begin{tabular}{lcccc}
\hline Methods & $\theta_{1}$ & $\theta_{2}$ & $\theta_{3}$ & $\theta_{4}$ \\
\hline BetP & 0.850 & 0.050 & 0.050 & 0.050 \\
PFT & 0.625 & 0.125 & 0.125 & 0.125 \\
CuzzP & 0.850 & 0.050 & 0.050 & 0.050 \\
PrPl & 0.925 & 0.025 & 0.025 & 0.025 \\
PrBel & 1.000 & 0.000 & 0.000 & 0.000 \\
PrNPl & 0.625 & 0.125 & 0.125 & 0.125 \\
PraPl & 0.925 & 0.025 & 0.025 & 0.025 \\
PropBelP & 1.000 & 0.000 & 0.000 & 0.000 \\
PropPlP & 0.925 & 0.025 & 0.025 & 0.025 \\
This paper & 0.985 & 0.005 & 0.005 & 0.005 \\
\hline
\end{tabular}

In the methods of PFT and PrNPl, $P\left(\theta_{1}\right)=0.625<$ $m\left(\theta_{1}\right)=0.8$, which is obviously unreasonable. Because the BPA of $\theta_{1} \cup \theta_{2} \cup \theta_{3} \cup \theta_{4}$ should not be assigned to $\theta_{1}$ completely, the results of PrBel and PropBelP are also unreasonable. In new method, $P\left(\theta_{1}\right)=0.985$ is far larger than $P\left(\theta_{2}\right)=$ $P\left(\theta_{3}\right)=P\left(\theta_{4}\right)=0.005$, which shows that new method is more reasonable and helpful to make decision than other methods.

2.4. Fusing the BPA. The navigation safety of underwater vehicles is affected by various ocean environment factors. If one factor makes underwater vehicles dangerous and others make underwater vehicles very safe, underwater vehicles should be in dangerous condition. Therefore, the BPA of each factor needs to be preprocessed before fusing to increase the weight of factor that makes underwater vehicles dangerous, which is consistent with the concept of super-standard weight. So the super-standard weight is used to deal with the BPA of each factor, and then a weighted average BPA is computed by the weight. Finally, D-S combination rule is used to obtain evaluation result through the former weighted average BPA.

The literature [35] gave a method to determine the superstandard weight according to the membership degree:

$$
\omega_{i}^{\prime}=\sum_{j=1}^{N} j r_{i j}
$$

where $N$ is the number of evaluation grades and $r_{i j}$ is the membership degree of $i$ th factor on $j$ th evaluation grade.

Definition 2. Here the BPA $m_{i, n}$ of $i$ th factor on $n$th evaluation grade $e_{n}$ is used to replace $r_{i j}$ :

$$
\omega_{i}^{\prime \prime}=\sum_{n=1}^{N} n m_{i, n}
$$

TABLE 4: Evaluation grades and quantitative intervals.

\begin{tabular}{lcc}
\hline Evaluation grade & Evaluation interval $(x)$ & Average value \\
\hline VS & $0 \leq x<20$ & 10 \\
MS & $20 \leq x<40$ & 30 \\
GS & $40 \leq x<60$ & 50 \\
MD & $60 \leq x<80$ & 70 \\
VD & $80 \leq x \leq 100$ & 90 \\
\hline
\end{tabular}

Definition 3. The weight $\omega_{i}$ of each factor can be obtained by normalizing the $\omega_{i}^{\prime \prime}$ :

$$
\omega_{i}=\frac{\omega_{i}^{\prime \prime}}{\sum_{i=1}^{k} \omega_{i}^{\prime \prime}}
$$

where $k$ is the number of factors.

Definition 4. The weighted average BPA $\bar{m}$ is acquired by using the weighted average method to deal with the BPA of each factor.

The weight of each factor and their BPAs can be written as in matrices $A$ and $B$ :

$$
\begin{gathered}
A=\left[\begin{array}{llll}
\omega_{1} & \omega_{2} & \cdots & \omega_{k}
\end{array}\right] \\
B=\left[\begin{array}{cccc}
m_{1}\left(e_{1}\right) & m_{1}\left(e_{2}\right) & \cdots & m_{1}\left(e_{N}\right) \\
m_{2}\left(e_{1}\right) & m_{2}\left(e_{2}\right) & \cdots & m_{2}\left(e_{N}\right) \\
\cdots & \cdots & \cdots & \cdots \\
m_{k}\left(e_{1}\right) & m_{k}\left(e_{2}\right) & \cdots & m_{k}\left(e_{N}\right)
\end{array}\right] .
\end{gathered}
$$

So $\bar{m}$ can be defined as

$$
\begin{aligned}
\bar{m} & =A \cdot B \\
= & {\left[\begin{array}{llll}
\omega_{1} & \omega_{2} & \cdots & \omega_{k}
\end{array}\right] } \\
& =\left[\begin{array}{cccc}
m_{1}\left(e_{1}\right) & m_{1}\left(e_{2}\right) & \cdots & m_{1}\left(e_{N}\right) \\
m_{2}\left(e_{1}\right) & m_{2}\left(e_{2}\right) & \cdots & m_{2}\left(e_{N}\right) \\
\cdots & \cdots & \cdots & \cdots \\
m_{k}\left(e_{1}\right) & m_{k}\left(e_{2}\right) & \cdots & m_{k}\left(e_{N}\right)
\end{array}\right] \\
= & {\left[\begin{array}{llll}
m_{1} & m_{2} & \cdots & m_{N}
\end{array}\right] . }
\end{aligned}
$$

At last, the evaluation result $C=\left[\begin{array}{lllll}r_{1} & r_{2} & r_{3} & r_{4} & r_{5}\end{array}\right]$ can be acquired by using $k-1$ times D-S combination rule to fuse $\bar{m}$.

2.5. Obtaining the Safety Situation Index. Safety situation should be a comprehensive description about each evaluation grade. Therefore, in order to make evaluation result of ocean environment safety situation more intuitive, the evaluation result needs to be disposed to obtain a rational and clear safety situation index. Here evaluation grades are quantized with different quantitative intervals, set $\{\mathrm{VS}, \mathrm{MS}, \mathrm{GS}, \mathrm{MD}$, $\mathrm{VD}\}=\{0-19,20-39,40-59,60-79,80-100\}$, and take their average values: $\{10,30,50,70,90\}$. Evaluation grades and corresponding quantitative intervals are as shown in Table 4. 


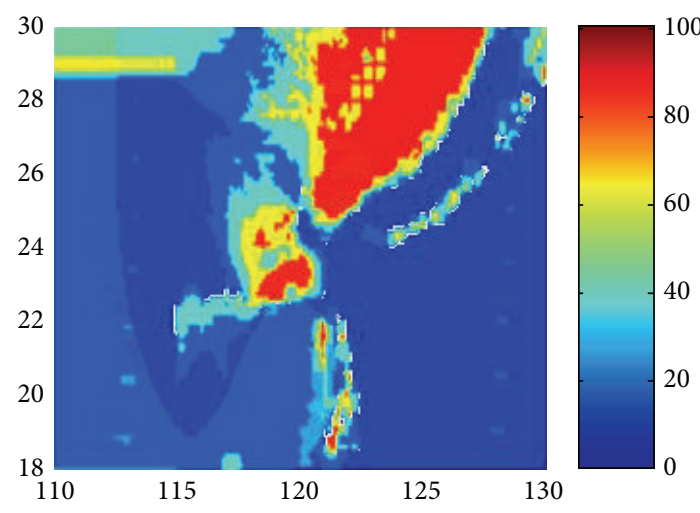

(a) $30 \mathrm{~m}$ depth

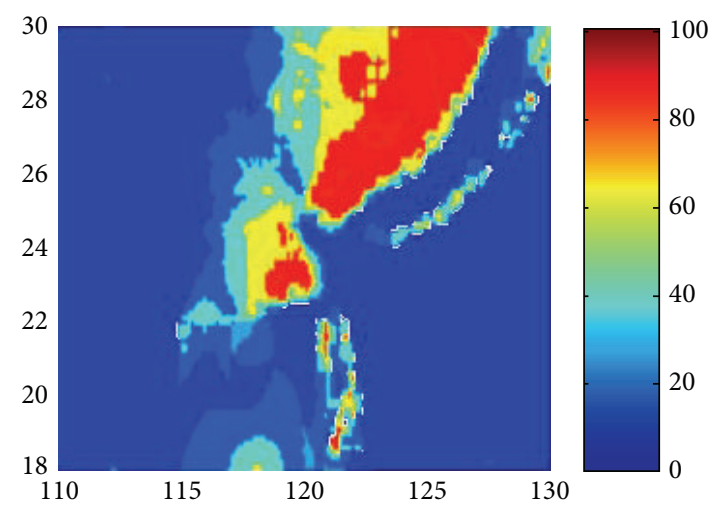

(b) $50 \mathrm{~m}$ depth

FIgURE 2: Safety situation (February).

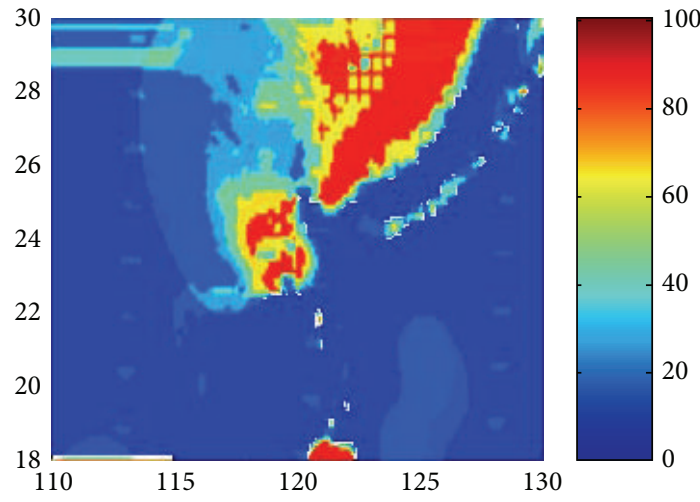

(a) $30 \mathrm{~m}$ depth

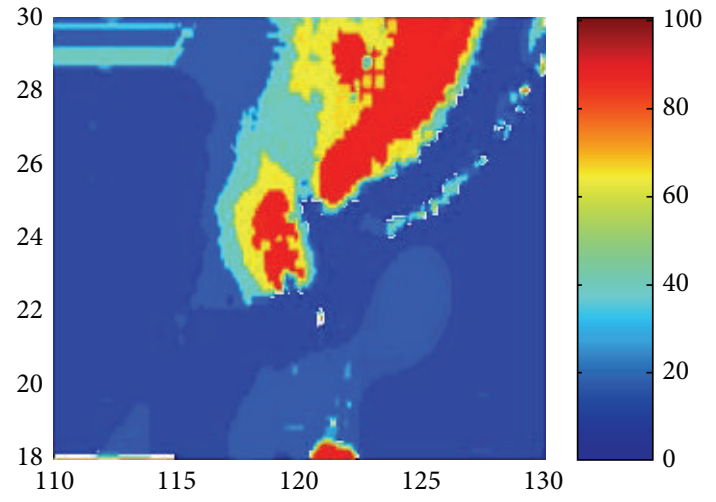

(b) $50 \mathrm{~m}$ depth

Figure 3: Safety situation (April).

The quantitative average values can be seen as a vector: $D=$ $\left[\begin{array}{lllll}10 & 30 & 50 & 70 & 90\end{array}\right]^{T}$.

Definition 5. Safety situation index is obtained by the product between the evaluation result and the average values. If the index is higher, the evaluation grade is more dangerous.

Namely, safety situation index SSI can be defined as:

$$
\begin{aligned}
\text { SSI } & =C \cdot D \\
& =\left[\begin{array}{lllll}
r_{1} & r_{2} & r_{3} & r_{4} & r_{5}
\end{array}\right] \cdot\left[\begin{array}{lllll}
10 & 30 & 50 & 70 & 90
\end{array}\right]^{T} .
\end{aligned}
$$

Obviously, SSI $\in[0,100]$.

\section{Results and Discussion}

In this section, simulations have been carried out aiming at validating the performance of evaluation method. According to the existing data of National Marine Data and Information Service, the analyzed data of ocean current, the forecasting data of tide current, and the statistical data of pycnocline are chosen, resolution is $0.125^{\circ}$, depth is $30 \mathrm{~m}$ and $50 \mathrm{~m}$, month is February, April, June, August, October, and December, longitude ranges from $110^{\circ} \mathrm{E}$ to $130^{\circ} \mathrm{E}$, and latitude ranges from $18^{\circ} \mathrm{N}$ to $30^{\circ} \mathrm{N}$. Since the existing data are all too small to fully describe the safety situation, in order to better illustrate the effectiveness of the proposed method, the existing data are magnified. The results can be seen in Figures 2-7. The different colors represent the different safety situation indexes, and horizontal axis represents longitude, while vertical axis represents latitude in each figure.

From results in Figures 2-7, it is obvious that the influence of ocean environment on underwater navigation safety has regional characteristics and seasonal characteristics. Safety situation indexes of various depths are different at the same position in the same month. Safety situation index is larger in some regions, and it is smaller in other regions. With the change of seasons, safety situation index also varies. The reason is that the values of ocean environment factors are various in different seasons. That is to say, ocean environment factors have seasonal characteristics. From the BPAs of ocean environment factors, it is easy to find that tide current plays a leading role in evaluating safety situation. With the change of months, the impact is also different. As can be seen from Figures 2 and 3, most areas are very dangerous, because the values of tide current in these areas are lager in February and April. However, tide current has a little impact on safety situation in June, August, October, and December. In Figures 


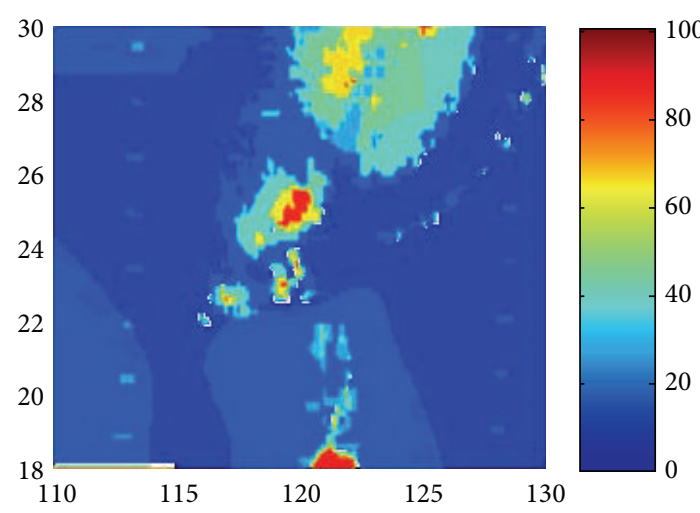

(a) $30 \mathrm{~m}$ depth

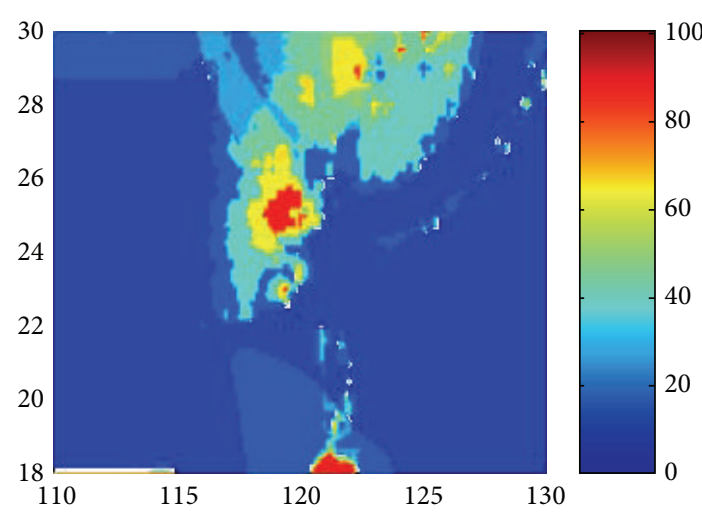

(b) $50 \mathrm{~m}$ depth

FIGURE 4: Safety situation (June).

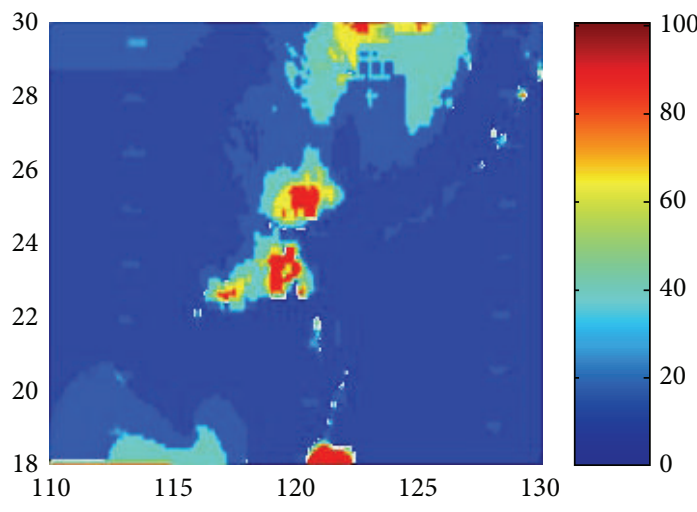

(a) $30 \mathrm{~m}$ depth

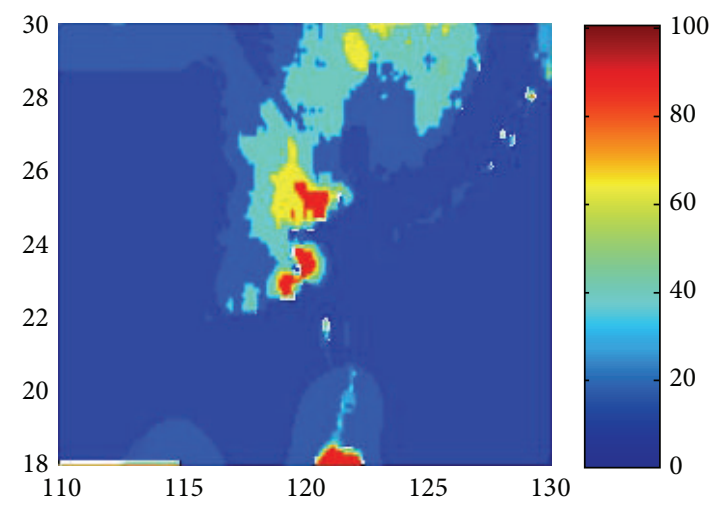

(b) $50 \mathrm{~m}$ depth

Figure 5: Safety situation (August).

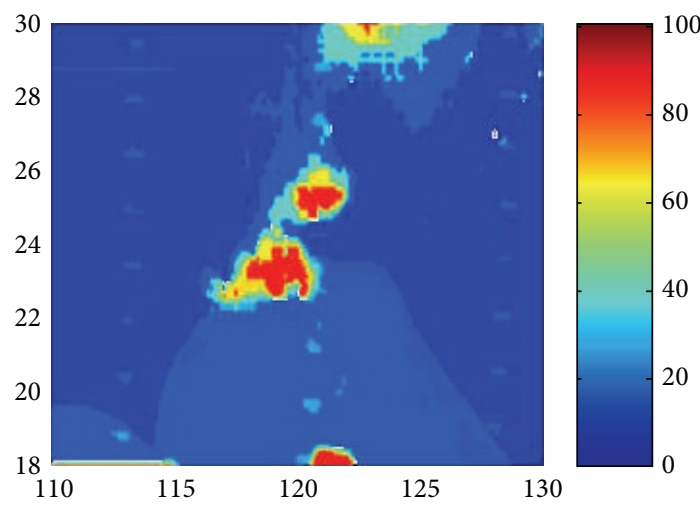

(a) $30 \mathrm{~m}$ depth

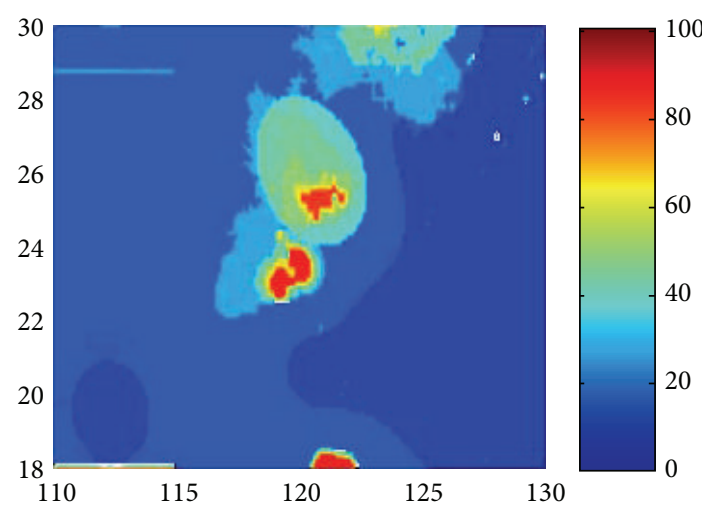

(b) $50 \mathrm{~m}$ depth

FIGURE 6: Safety situation (October).

4,5 , and 6, there are few "very dangerous" regions and "more dangerous" regions. In particular, they is almost none in Figure 7.

\section{Conclusions}

Ocean environment has a significant impact on underwater navigation safety. No matter how an underwater vehicle is carrying out the task or sailing, the influence of ocean environment on navigation safety needs to be closely grasped. Due to distinguished performance of D-S evidence theory on handling uncertainty information, it is quite befitting for evaluating underwater ocean environment safety situation. This theory is used to deal with ocean environment information to acquire evaluation result of underwater ocean environment safety situation in this paper. The simulation results 


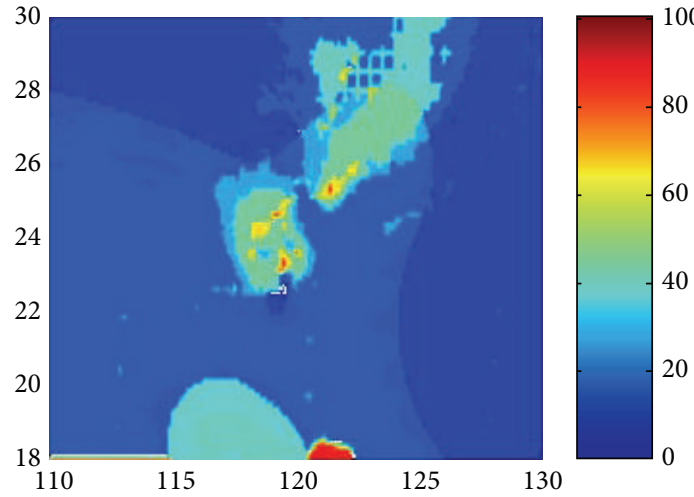

(a) $30 \mathrm{~m}$ depth

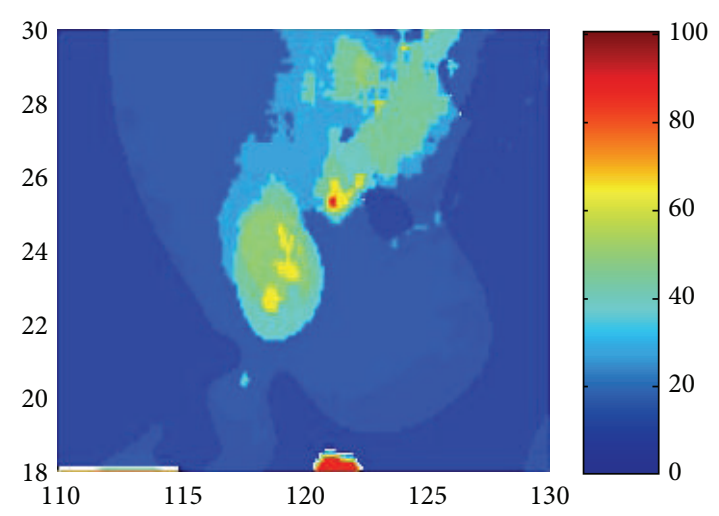

(b) $50 \mathrm{~m}$ depth

Figure 7: Safety situation (December).

validate that the method proposed is of great theoretical value and practical significance.

\section{Conflict of Interests}

The authors declare that there is no conflict of interests regarding the publication of this paper.

\section{Acknowledgments}

This paper is supported by the National Natural Science Foundation of China (Projects nos. 51379049 and 51109045), the Fundamental Research Funds for the Central Universities (Projects nos. HEUCFX41302 and HEUCF041410), the Scientific Research Foundation for the Returned Overseas Chinese Scholars (Heilongjiang Province, no. LC2013C21), and the Young College Academic Backbone of Heilongjiang Province (no. 1254G018).

\section{References}

[1] Y. R. Xu and K. Xiao, “Technology development of autonomous ocean vehicle," Acta Automatica Sinica, vol. 33, no. 5, pp. 518521, 2007.

[2] Y. Gao, B. Yin, Z. Q. Wei, F. X. Gong, and X. P. Ji, "On-line realtime path planning for underwater vehicle in dynamic uncertain environment," Periodical of Ocean University of China, vol. 43, no. 12, pp. 106-110, 2013.

[3] D. Q. Zhu, W. C. Li, and M. Z. Yan, "Map building for underwater vehicle based on D-S information fusion algorithm," System Simulation Technology, vol. 8, no. 3, pp. 181-186, 2012.

[4] X. Li and D. Q. Zhu, "Path planning for autonomous underwater vehicle based on artificial potential field method," Journal of Shanghai Maritime University, vol. 31, no. 2, pp. 35-39, 2010.

[5] N. K. Yilmaz, C. Evangelinos, P. F. J. Lermusiaux, and N. M. Patrikalakis, "Path planning of autonomous underwater vehicles for adaptive sampling using mixed integer linear programming," IEEE Journal of Oceanic Engineering, vol. 33, no. 4, pp. 522-537, 2008.

[6] I. Tena Ruiz, S. de Raucourt, Y. Petillot, and D. M. Lane, "Concurrent mapping and localization using sidescan sonar," IEEE Journal of Oceanic Engineering, vol. 29, no. 2, pp. 442-456, 2004.
[7] A. Alvarez, A. Caiti, and R. Onken, "Evolutionary path planning for autonomous underwater vehicles in a variable ocean," IEEE Journal of Oceanic Engineering, vol. 29, no. 2, pp. 418-429, 2004.

[8] A. P. Dempster, "Upper and lower probabilities induced by a multi-valued mapping," The Annals of Mathematical Statistics, vol. 38, pp. 325-339, 1967.

[9] G. Shafer, A Mathematical Theory of Evidence, Princeton University Press, Princeton, NJ, USA, 1976.

[10] Y. Leung, N.-N. Ji, and J.-H. Ma, "An integrated information fusion approach based on the theory of evidence and group decision-making," Information Fusion, vol. 14, no. 4, pp. 410422, 2013.

[11] H. Luo, S. L. Yang, X. J. Hu, and X. X. Hu, "Agent oriented intelligent fault diagnosis system using evidence theory," Expert Systems with Applications, vol. 39, no. 3, pp. 2524-2531, 2012.

[12] B. Li and F.-W. Pang, "An approach of vessel collision risk assessment based on the D-S evidence theory," Ocean Engineering, vol. 74, pp. 16-21, 2013.

[13] J. P. Yang, H.-Z. Huang, L.-P. He, S.-P. Zhu, and D. W. Wen, "Risk evaluation in failure mode and effects analysis of aircraft turbine rotor blades using Dempster-Shafer evidence theory under uncertainty," Engineering Failure Analysis, vol. 18, no. 8, pp. 2084-2092, 2011.

[14] R. P. Srivastava, T. J. Mock, and L. Gao, "The Dempster-Shafer theory: an introduction and Fraud risk assessment illustration," Australian Accounting Review, vol. 21, no. 3, pp. 282-291, 2011.

[15] B. Y. Kang, Y. Li, Y. Deng, Y. J. Zhang, and X. Y. Deng, "Determination of basic probability assignment based on interval numbers and its application," Acta Electronica Sinica, vol. 40, no. 6, pp. 1092-1096, 2012.

[16] J. Y. Xiao, M. M. Tong, C. J. Zhu, and X. L. Wang, "Basic probability assignment construction method based on generalized triangular fuzzy number," Chinese Journal of Scientific Instrument, vol. 33, no. 2, pp. 429-434, 2012.

[17] T. Denœux and M.-H. Masson, "EVCLUS: evidential clustering of proximity data," IEEE Transactions on Systems, Man, and Cybernetics Part B: Cybernetics, vol. 34, no. 1, pp. 95-109, 2004.

[18] X. Guan, Y. He, and X. Yi, "Attribute measure recognition approach and its applications to emitter recognition," Science in China, Series F: Information Sciences, vol. 48, no. 2, pp. 225-233, 2005.

[19] W. Jiang, A. Zhang, and Q. Yang, "Fuzzy approach to construct basic probability assignment and its application in multi-sensor 
data fusion systems," Chinese Journal of Sensors and Actuators, vol. 21, no. 10, pp. 1717-1720, 2008.

[20] Y. Deng, W. Jiang, X. B. Xu, Q. Li, and D. Wang, “Determinging BPA under uncertainty environments and its application in data fusion," Journal of Electronics, vol. 26, no. 1, pp. 13-17, 2009.

[21] F. Han, W. H. Yang, and X. G. Yuan, "Evidence theory information fusion method based on fuzzy set," Control and Decision, vol. 25, no. 3, pp. 449-452, 2010.

[22] N. Li, C.-W. Qu, D.-F. Ping, and F. Su, "Emitter identification based on distributed sensors information fusion," Control and Decision, vol. 25, no. 12, pp. 1793-1798, 2010.

[23] W. Jiang and S. Wu, "Multi-data fusion fault diagnosis method based on SVM and evidence theory," Chinese Journal of Scientific Instrument, vol. 31, no. 8, pp. 1738-1743, 2010.

[24] P. Smets and R. Kennes, "The transferable belief model," Artificial Intelligence, vol. 66, no. 2, pp. 191-234, 1994.

[25] B. R. Cobb and P. P. Shenoy, "On the plausibility transformation method for translating belief function models to probability models," International Journal of Approximate Reasoning, vol. 41, no. 3, pp. 314-330, 2006.

[26] P.-D. Xu, D.-Q. Han, and Y. Deng, "An optimal transformation of basic probability assignment to probability," Acta Electronica Sinica, vol. 39, no. 3A, pp. 121-125, 2011.

[27] L. Hu, Y. He, X. Guan, Y. Deng, and D. Han, "A new probabilistic transformation in generalized power space," Chinese Journal of Aeronautics, vol. 24, no. 4, pp. 449-460, 2011.

[28] W.-Q. Wang, Y.-J. Zhao, J. Huang, and T. Lai, “Transformation of basic probability assignment to probability based on uncertainty degree," Control and Decision, vol. 28, no. 8, pp. 1214-1218, 2013.

[29] J. J. Sudano, "Pignistic probability transforms for mixs of lowand high probability events," in Proceedings of the 4th International Conference on Information Fusion, pp. 23-27, Montreal, Canada, 2001.

[30] W. Jiang, C. C. Wu, J. Jia, and Z. J. Li, "A probabilistic transformation of basic probability assignment (BPA) in D-S evidence theory," Journal of Northwestern Polytechnical University, vol. 31, no. 2, pp. 295-299, 2013.

[31] L. F. Hu, Y. He, X. Guan, D. Q. Han, and Y. Deng, "New probabilistic transformation of imprecise belief structure," Journal of Systems Engineering and Electronics, vol. 22, no. 5, pp. 721-729, 2011.

[32] J. J. Sudano, "Yet another paradigm illustrating evidence fusion (YAPIEF)," in Proceedings of the 9th International Conference on Information Fusion (FUSION '06), July 2006.

[33] J. Dezert and F. Smarandache, "A new probabilistic transformation of belief mass assignment," in Proceedings of the 11th International Conference on Information Fusion, pp. 1-8, Cologne, Germany, July 2008.

[34] M. Daniel, "Consistency of probabilistic transformations of belief functions," in Proceedings of the 10th International Conference on Information Processing and Managment of Uncertainty in Knowledge-Based Systems (IPMU '04), pp. 1135-1142, Perugia, Italy, 2004.

[35] J. H. Shen, X. Y. Fu, and Y. X. Zhao, "Improvement of the fuzzy comprehensive evaluation model," Fuzzy Systems and Mathematics, vol. 25, no. 3, pp. 127-132, 2011. 

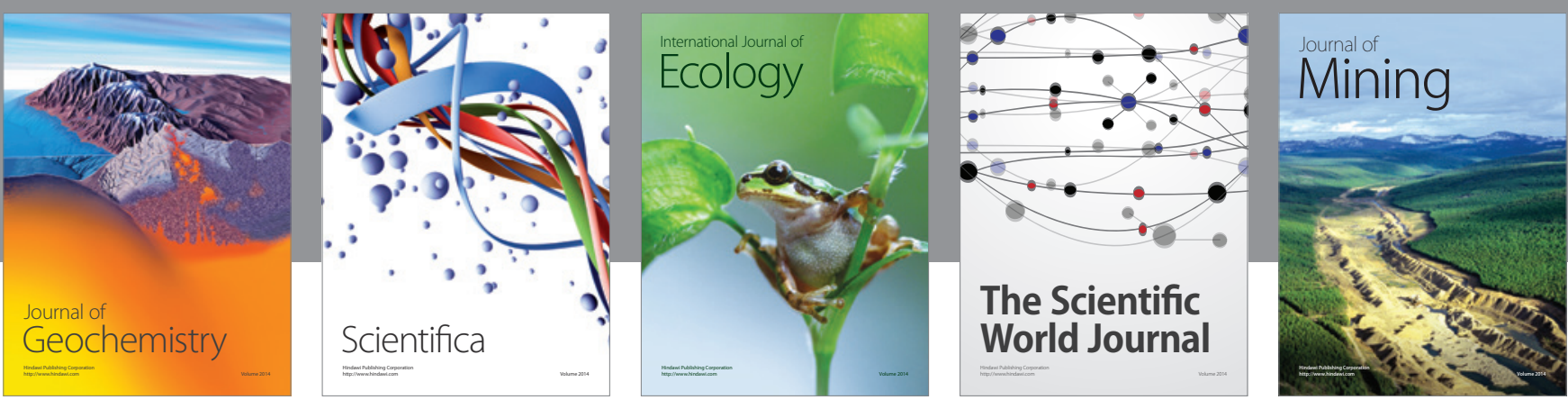

The Scientific World Journal
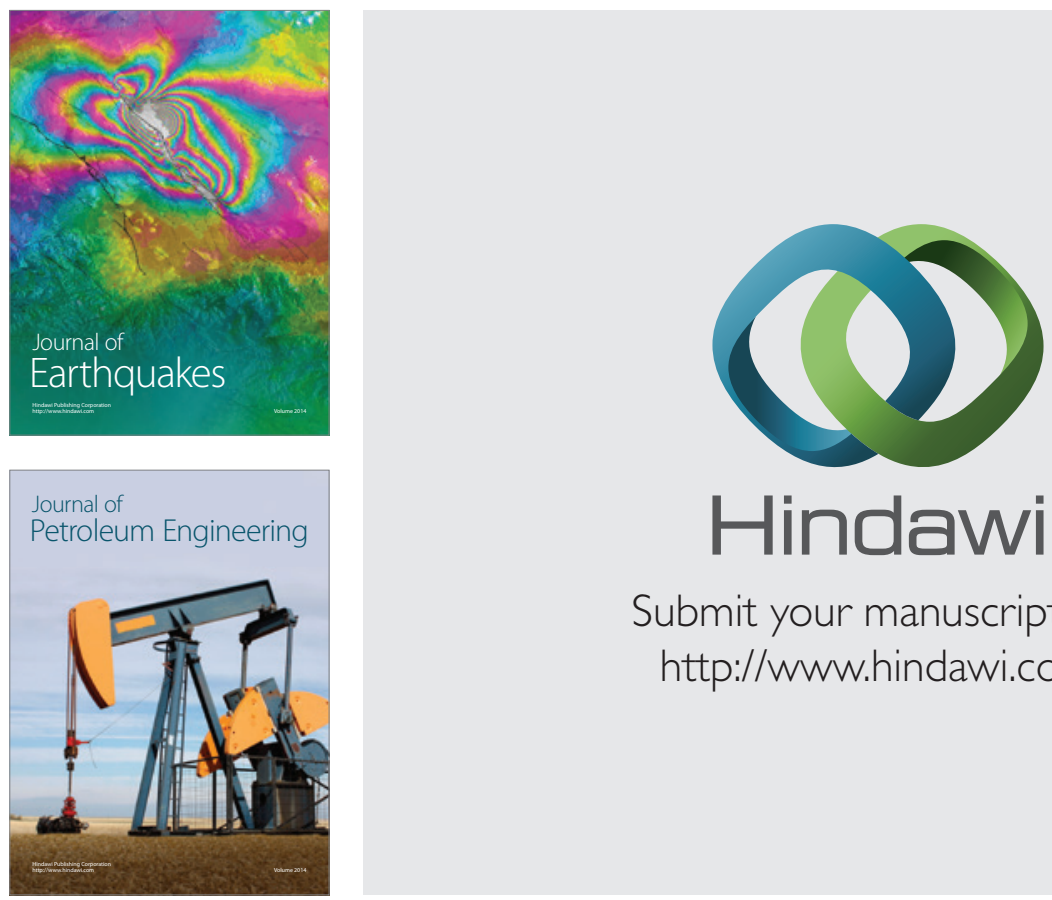

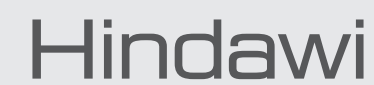

Submit your manuscripts at

http://www.hindawi.com
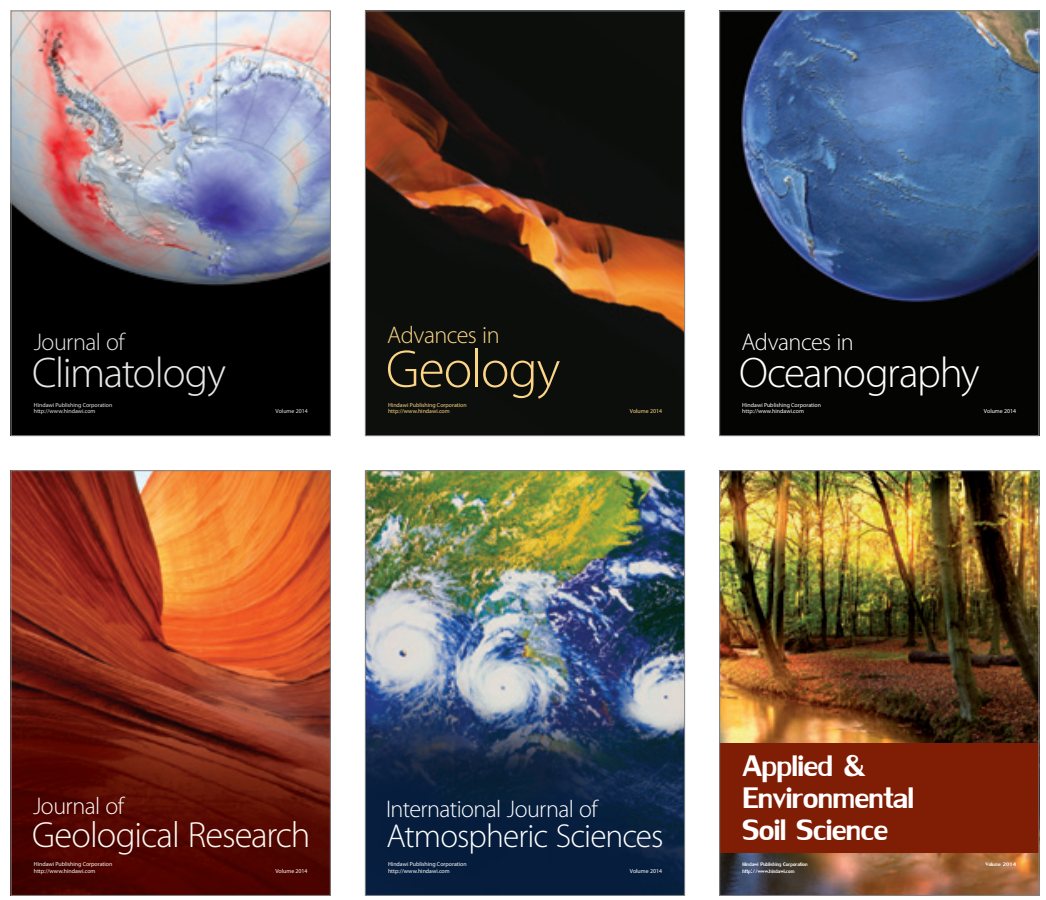
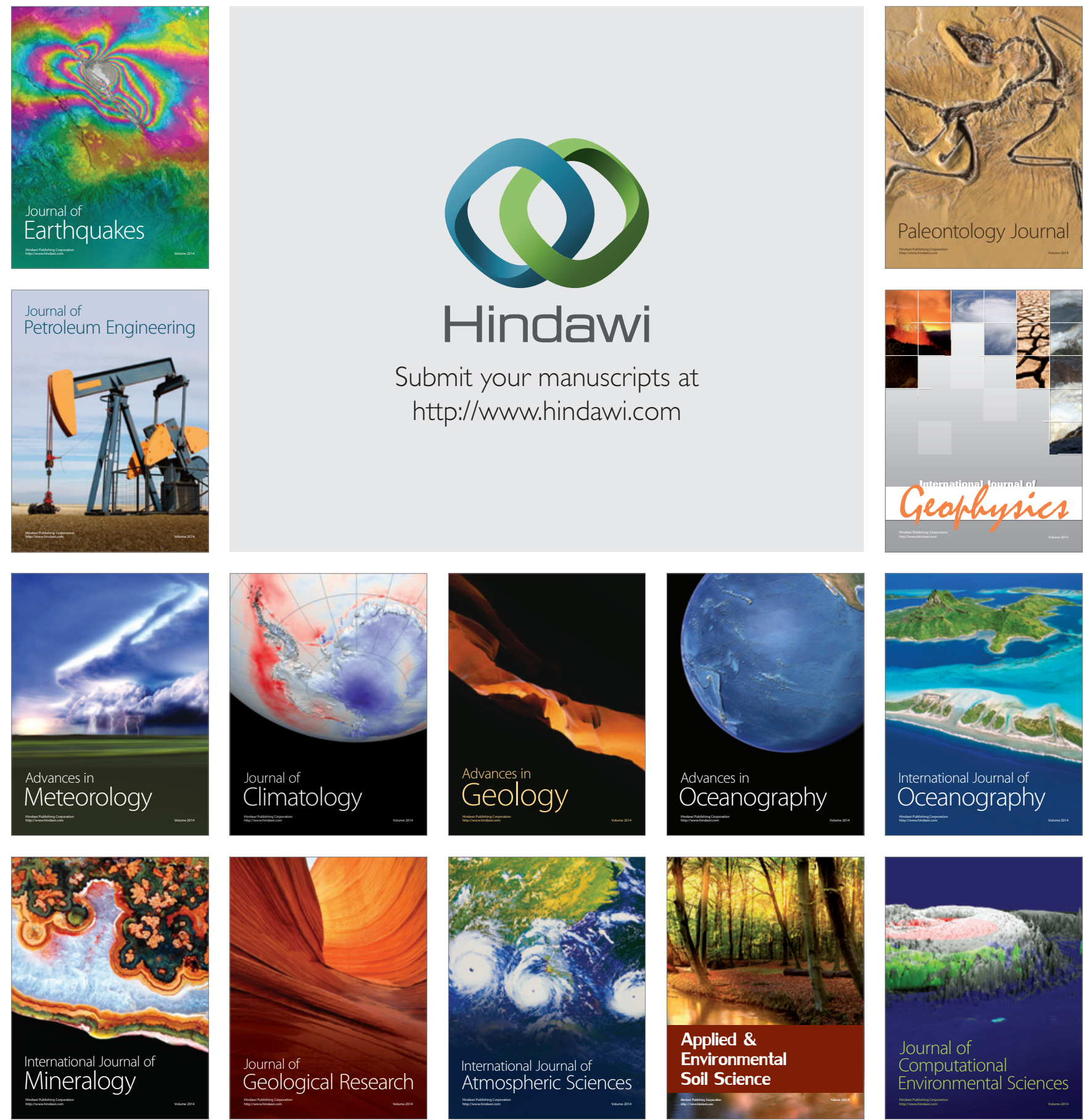